\title{
Interferometric Readout for a Monolithic Accelerometer, towards the $\mathrm{fm} / \sqrt{\mathrm{Hz}}$
}

\author{
J.V. van Heijningen* \\ National Institute for Subatomic Physics Nikhef, Amsterdam, The Netherlands \\ E-mail: jvnheijn@nikhef.nl
}

\section{A. Bertolini}

National Institute for Subatomic Physics Nikhef, Amsterdam, The Netherlands

E-mail: alberto@nikhef.nl

\section{Rabeling}

National Institute for Subatomic Physics Nikhef, Amsterdam, The Netherlands

E-mail: d.rabeling@nikhef.nl

\section{J.F.J. van den Brand}

VU University, Amsterdam, The Netherlands

E-mail: jo@nikhef.nl

\begin{abstract}
The Virgo Gravitational Wave Observatory (Cascina, Italy) has encountered problems with scattered light from vibrating, out-of-vacuum, sensing optics coupling back into the interferometer resulting in lower sensitivies. The upcoming upgrade for this detector necessitates for scattered light mitigation. An optical bench houses the optics and this bench has to be seismically isolated as well, in a similar way as the mirrors of the interferometer have to be decoupled from the Earth's movement. Nikhef has designed and built the compact isolator to do this, called MultiSAS, but to measure its residual motion in full assembly and its isolation performance, no (commercial) sensor is available that is good enough to actually measure it. A novel vibration sensor is built at Nikhef by using an interferometric readout for a horizontal monolithic accelerometer, which should be able to measure in the vicinity of the $\frac{f m}{\sqrt{H z}}$ regime. This accelerometer is compact for its performance and vacuum compatible. Current results show lowest noise levels around $70 \frac{\mathrm{fm}}{\sqrt{\mathrm{Hz}}}$, 1.5 orders of magnitude too high, for which two possible reasons are suspected.
\end{abstract}

Technology and Instrumentation in Particle Physics 2014,

2-6 June, 2014

Amsterdam, the Netherlands

\footnotetext{
* Speaker.
} 


\section{Introduction}

The Virgo Gravitational Wave Observatory has started its science runs in 2007 and is currently undergoing an upgrade to Advanced Virgo. The angles between the main mirrors of the interferometer (IFO) are aligned by an automatic alignment system to levels of nrad rms. Previously, this was done by splitting off (pick-off) a small portion of the main light beam and, through a viewport, performing optical sensing on a bench, which was not seismically isolated. Scattered light from the optics coupled back into the interferometer and degraded sensitivities, which was solved by anti-reflection coatings. In order to achieve the target sensitivity for the upgrade, the bench with the sensing optics that monitor the beam positions for this alignment is seismically isolated. The isolator to do this has to be able to isolate an optical bench to the amplitude spectral density (ASD) level of $10^{-12} \frac{\mathrm{m}}{\sqrt{\mathrm{Hz}}}$ for translational motion and $10^{-15} \frac{\mathrm{rad}}{\sqrt{\mathrm{Hz}}}$ for angular motion. One can view an ASD as a spectral decomposition of a signal, where integration of such a curve results in the rms value of the signal. In Fig. 1(b), the isolator to do just that is shown: MultiSAS, a design by Nikhef in order to cope with the above requirements. From the scale visible from the observing physicist, the optical bench has typical dimensions of order a meter, so $10^{-15} \frac{\mathrm{rad}}{\sqrt{\mathrm{Hz}}}$ will be measured as $10^{-15} \frac{m}{\sqrt{H z}}$ close to the edges in the angular degrees of freedom by a horizontal accelerometer.

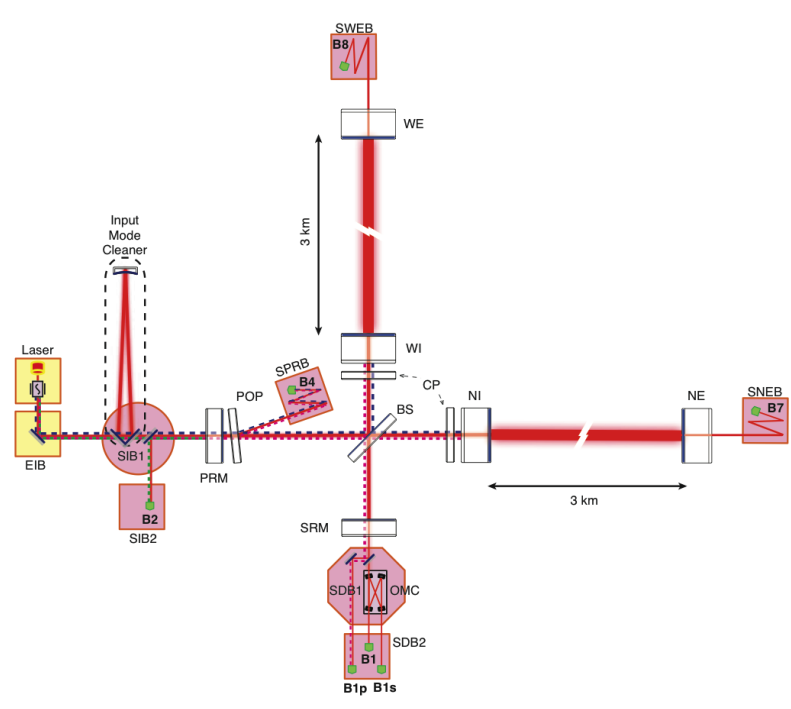

(a)

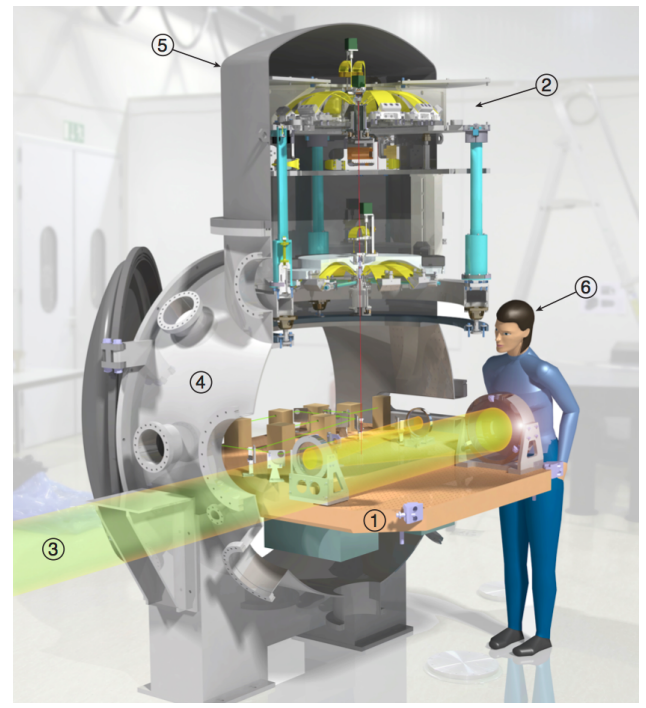

(b)

Figure 1: (a) The optical set-up for AdVirgo showing the interferometer with $3 \mathrm{~km}$ long arms. At the end of those arms, using the transmissive light of the mirrors, or picked off at various locations (pink squares, e.g. SNEB, in the picture), sensing is applied housed on optical bences seismically isolated by (b) the MultiSAS compact isolator

The stringent requirements for the residual motion of the optical bench suspended by MultiSAS necessitate a sensor that is able to measure and/ or place a more stringent upper limit on this motion. An inertial sensor with a broadband sensitivity in the vicinity of the $\frac{f m}{\sqrt{H z}}$ regime is needed for such a measurement. Another goal of such a sensor is a total transfer function (TF) measurement from ground to suspended bench. At the time of need for these measurements, available used or commercial sensors were not sensitive enough and thus the decision was made at Nikhef to 
alter an already used concept, the horizontal monolithic accelerometer described in A. Bertolini's thesis [1].

\section{Monolithic accelerometer}

A vibration sensor such as an accelerometer, in its most basic form, is a suspended mass where the movement of the mass with respect to some reference frame is a measure for the acceleration the mass is undergoing. The reference frame moves and, because of its inertia, the mass lags this movement. If one is able to determine the movement of the mass with respect to the reference frame, mechanical vibrations can be measured.

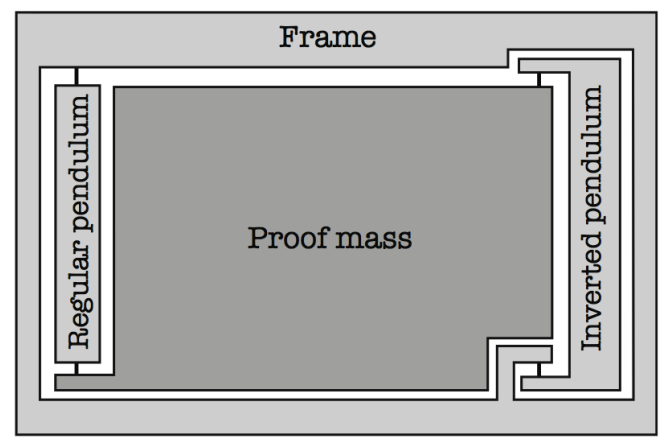

Figure 2: Mechanical set-up of the monolothic accelerometer

Fig. 2 shows the mechanics of the monolithic accelerometer used in this sensor. The so-called proof mass is suspended by a regular pendulum and, in a clever way, by an inverted one. This is done to lower the overall resonance frequency of the system, without having a long pendulum. The inverted pendulum acts as a broom on a hand in a sense that the force moving the mass away from the equilibrium position increases as the mass moves away from this equilibrium position; an inverted pendulum introduces a negative spring constant. Together with the regular pendulum, the spring constants of the overall system are added and the system can be tuned to very low resonance frequencies; for this sensor it is at $0.55 \mathrm{~Hz}$. The upper limit of the bandwidth of the sensor is about $100 \mathrm{~Hz}$. This is the first internal resonance of the mechanics; the two legs of the pendulums start becoming floppy at these frequencies. The accelerometer is made from a high strength aluminium alloy 7075-T6, for its high yield strength of $500 \mathrm{MPa}$.

The combination of the regular pendulum and inverted pendulum allows for the flexures of the inverted pendulum to be loaded in tension. This allows for much thinner flexures, which lowers the amount of energy that can be stored in the flexures and this lowers the suspension thermal dissipation noise. This noise described by Callen and Welton postulates there is a relation between the response of a driven dissipative system and the spontaneous fluctuations of a generalized variable, i.e. the position, of the system in equilibrium [2]. It results in the Brownian motion in the flexures of both pendulums giving rise to noise in the proof mass' motion.

\section{Interferometric readout}

In Fig. 3, a particular set-up for the readout of the movement of the proof mass is depicted, 
where a small balanced IFO [3] is used to determine the mass' position. Both arms of the IFO are read out, matched in magnitude and subtracted in order to lose common mode noise, such as intensity fluctuations in the laser power. This signal is used as an error signal in a feedback loop (FBL) in which the actuator is the Voice Coil (VC).

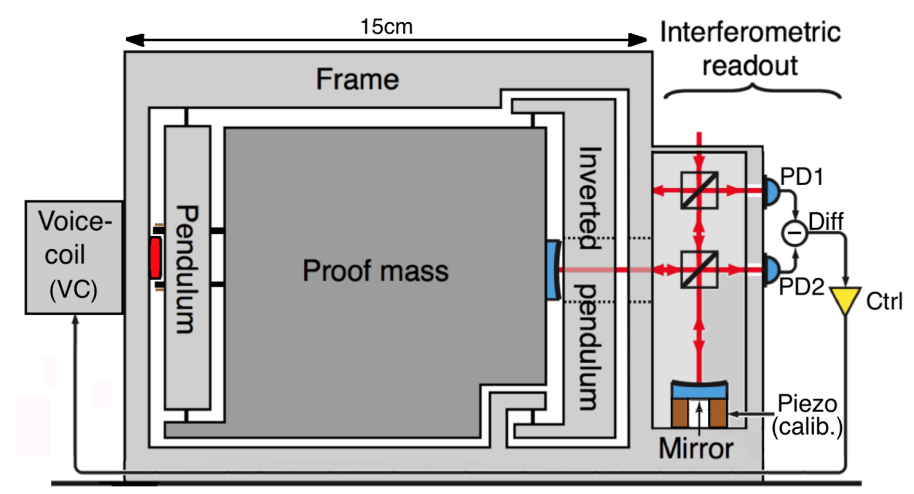

Figure 3: Opto-mechanical set-up, where the position of the proof mass is read out by a table top IFO of which both arms are read out. These signals are subtracted to obtain a differential signal, which is fed to a controller at the input of a FBL. The output of this controller is fed to a coil magnet actuator (VC) and this keeps the mass in a position close to the lock-point. The signal fed to the voice coil can be taken as the sensor output

The lower beamsplitter (BS) and the two mirrors make up the classical Michelson IFO, where one mirror is attached to the proof mass and the other is on a piezoelectric crystal (piezo) for calibration reasons. The laser source used is NP Photonics' The Rock, which has a linewidth of $700 \mathrm{~Hz}$ ensuring non-dominant frequency noise in this IFO when the two arms are not of equal length. By physically locking the proof mass, the piezo can be used to apply known translations to one mirror. By knowing the $\lambda$ of the light used $(1550 \mathrm{~nm})$ and inspecting the output sinusoids when applying a linear ramp to the piezo, a conversion factor is determined between output voltages of the differential signal and mirror displacements. This is shown in Fig 4(a), where another reason for subtraction becomes apparent. A ramp signal is fed to the piezo causing it to move the mirror and interference patterns start to arise on both photodiodes (PD). PD2 has roughly twice the amplitude here as PD1 as the PD1 beam goes through another BS. Matching and subtracting these PD signals results in the differential signal, which has the linear part of the sinusoid around $0 \mathrm{~V}$. This is a lockpoint where the controller can lock and thus ensure linear output of the sensor. At this lock-point, a slope of $21.52 \frac{n m}{V}$ is determined.

To see if the subtraction of common mode intensity noise works properly, one of the arms of the IFO is blocked and just the DC voltages are read out. The differential signal is then tuned to 0 $\mathrm{V}$, using a variable resistor (potmeter) in the differential amplifier. In Fig 4(b), such a noise measurement, converted from $\frac{V}{\sqrt{H z}}$ to $\frac{m}{\sqrt{H z}}$ by the previously determined conversion factor, is shown. From the voltages that fall on the PDs, the power can be determined and, with these wattages, the shot noise limit can be calculated using

$$
i_{s n, P D}=\sqrt{2 e I_{P D}}=\sqrt{2 e \rho P_{P D}},
$$




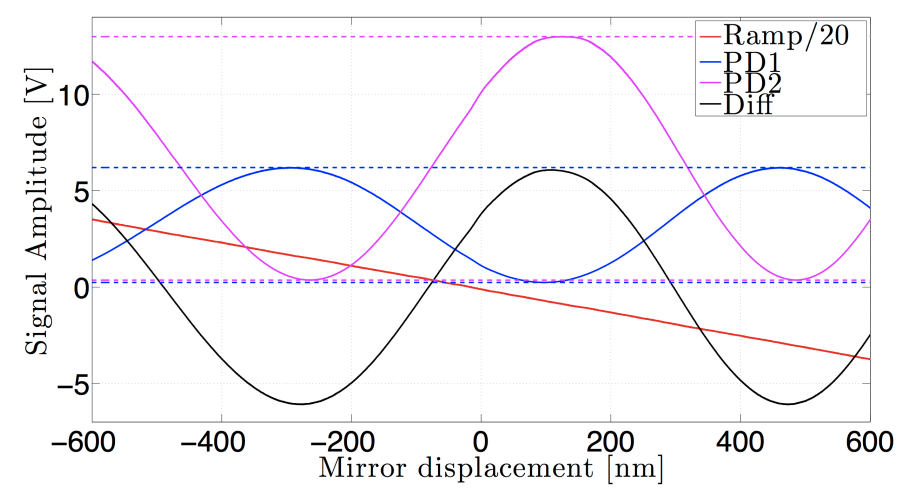

(a)

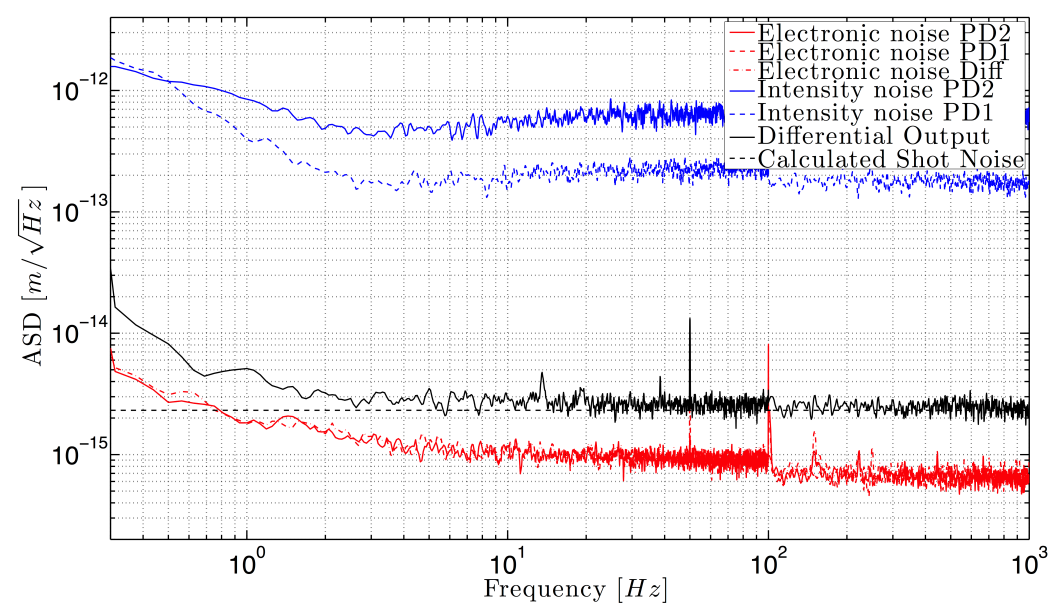

(b)

Figure 4: (a) Readout signals of the IFO when applying a linear driving signal to the piezo. The units on the horizontal axis are determined by the knowledge of the distances between minimum and maximum of a fringe, which is a quarter of a wavelength, and (b) ASD in $\mathrm{m} / \sqrt{\mathrm{Hz}}$ of different noises in the different channels of the interferometric readout

where $e$ is the elementary charge and $\rho$ is the responsivity in $\mathrm{A} / \mathrm{W}$ of the PD. The power of the beams on the PDs in the previously shown noise measurement is $P_{P D 1}=70 \mu \mathrm{W}$ and $P_{P D 2}=180 \mu \mathrm{W}$ and, knowing the resistances used in the amplifiers of the readout circuit, one can determine the shot noise limit. Conversion of that limit to $\frac{m}{\sqrt{H z}}$ leads to the 2-3 $\frac{f m}{\sqrt{H z}}$ visible in Fig. 4(b). From 10 $\mathrm{Hz}$ onwards, the subtraction of the intensity noise is shot-noise limited.

When the interferometric readout is in operation, the FBL must ensure that the differential signal is kept around $0 \mathrm{~V}$ as much as possible. The larger this residual arm length imbalance becomes, the more the intensity noise subtraction breaks down.

In Fig. 5 the modelled noise budget is shown, where the two previously discussed noise sources, the suspension dissipation thermal noise and the shot noise, are dominant. The relative intensity noise (RIN) converges to the shot-noise from $3 \mathrm{~Hz}$ onwards, which is visible in the subtraction performance shown in Fig. 4(b). The non-dominant noises are all noises associated with the readout electronics. All the noise curves but the suspension thermal one, show a notch around $0.55 \mathrm{~Hz}$, the natural frequency of the accelerometer. This is because the mechanical response, or 


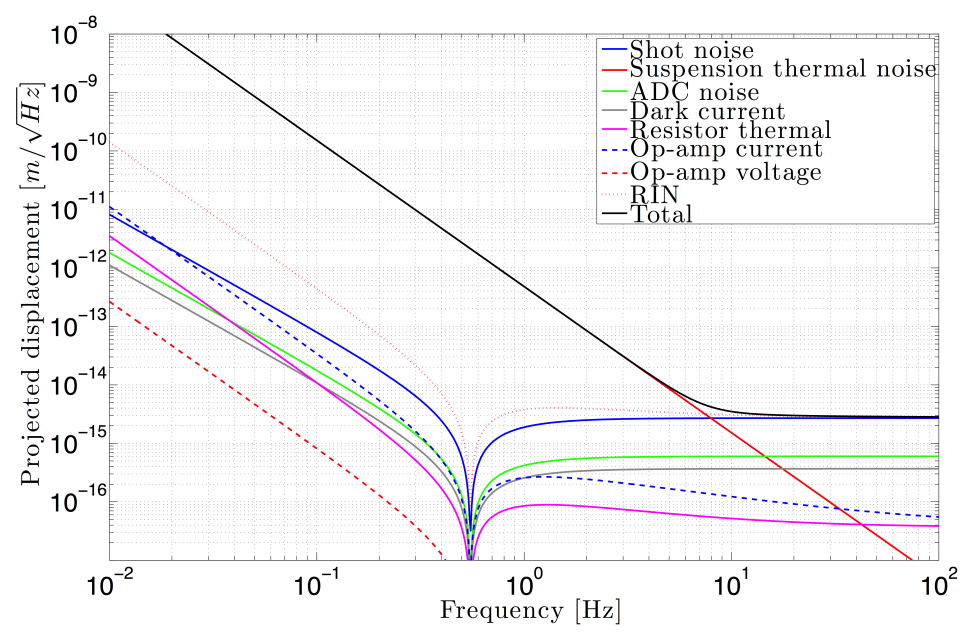

Figure 5: Noise budget of the suspended mass with an interferometric readout. Contributions of the (readout) electronics are expected lower than the two dominant noise sources: suspension thermal dissipation noise and the shot noise

TF, of the accelerometer has been divided out. The division is necessary as noises in the readout look at the relative movement of the proof mass, which in its turn is governed by the TF of the harmonic oscillator that is used. Below the resonance frequency, the relative movement of the proof mass is lower for excitations of the same amplitude and, logically, a noise in the readout has more effect on how precise such a smaller relative movement can be detected. Having a low natural frequency helps here as the noise curves of all the non-dominant noises would shift towards the right in this plot with the possibility of becoming dominant.

\section{Towards the $f m / \sqrt{H z}$}

Placing the sensor in vacuum on an optical bench suspended by the MultiSAS, as is shown in Fig 6, an environment where (part) of the sensor's noise floor is visible is found. The low frequency part is clouded by the mechanical modes of the MultiSAS $(0.08 \mathrm{~Hz}, 0.75 \mathrm{~Hz}$ and $1.8 \mathrm{~Hz})$, but from then onwards the tail of suspension dissipation noise and the shot-noise limited floor from $10 \mathrm{~Hz}$ onwards can be measured.

During the measurements done so far with the sensor, the controls of the MultiSAS have not been operational because of issues with the digital system. This has its effect on the residual bench motion and, because the optical bench is suspended by one rod in its centre of mass, couplings of this motion to angular modes is inevitable. The uncontrolled angular motion as well as the horizontal motion of the bench is measured by the sensor of which results are shown in Fig. 7.

This result shows a level arising from $40 \mathrm{~Hz}$ onwards of about $70 \frac{\mathrm{fm}}{\sqrt{\mathrm{Hz}}}$, which is roughly 1.5 orders of magnitude higher than the goal. Below $3 \mathrm{~Hz}$, the sensor is measuring the residual bench motion, with the $1.8 \mathrm{~Hz}$ mode of the isolator chain. A number of other peaks are apparent in the measurement. The peaks around 23 and $30 \mathrm{~Hz}$ are associated with the vacuum pump, $50 \mathrm{~Hz}$ is the electrical mains frequency and $60 \mathrm{~Hz}$ might be a resonance in MultiSAS' isolating chain. Monitoring the differential signal during this measurement shows deviations from $0 \mathrm{~V}$, because the 


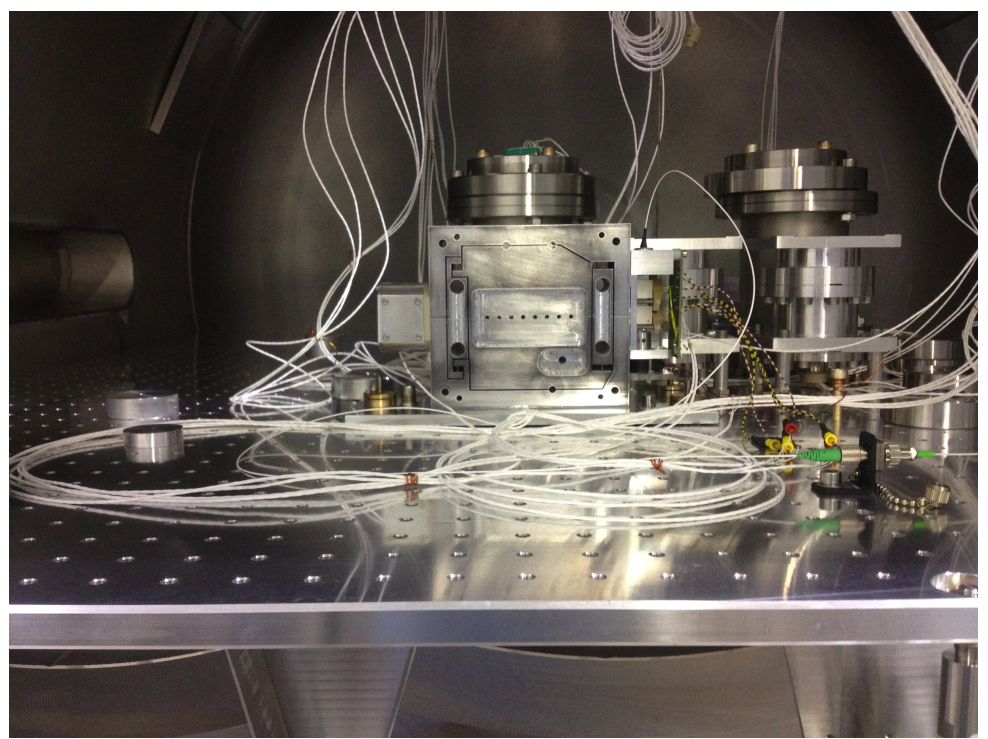

Figure 6: Photograph of the monolithic accelerometer with an interferometric readout on MultiSAS' optical bench. Other sensors in vacuum enclosures are also visible on the bench

control loop is not able to make the VC damp fully the coupling of all these motions to the proof mass. This means the intensity noise of the laser spoils the shot-noise limited noise floor.

Another possible reason for the goal not being obtained is polarization noise. The laser's light is brought to the sensor by use of a single mode fiber, as is visible in Fig. 6. During calibration of the sensor, upon bending the fiber the fringe visibility or contrast goes down. The fringe visibility is the ratio of the amplitude and average of the interference pattern (like PD1 and PD2 in Fig. 4(a)) and thus $100 \%$ if the minimum of such pattern is zero. A visibility of $97 \%$ is obtained and means that parts of the two interfering beams are not participating in the interference. Misalignment can cause this, but in the case of bending the fiber, polarization changes cause different transmissions and reflection coefficients in the BSs which cause imbalances in the amounts of photons in both arms of the IFO. If one arm of the IFO has more photons, some of them will not participate in the interference as there are simply not enough photons coming from the other arm. Mechanical vibrations can couple into the fiber and have a similar, but much smaller and varying effect causing noise in the readout.

\section{Conclusion and future work}

Development of the novel sensor is ongoing at Nikhef, with the current sensor noise floor around $70 \frac{f m}{\sqrt{H z}}$ from $40 \mathrm{~Hz}$ onwards. Two suspected reasons for the noise floor not to be around the modelled goal of 2-3 $\frac{\mathrm{fm}}{\sqrt{\mathrm{Hz}}}$ are the uncontrolled residual motion of the optical bench resulting in non-optimal subtraction of the laser's intensity noise and polarization noise.

The residual motion of the bench is to be controlled in horizontal and vertical by already installed controls, but for the angular control 4 sensor - actuator pairs have been installed at the bench level for horizontal (yaw) as well as vertical (pitch and roll) control. When these are operational, 


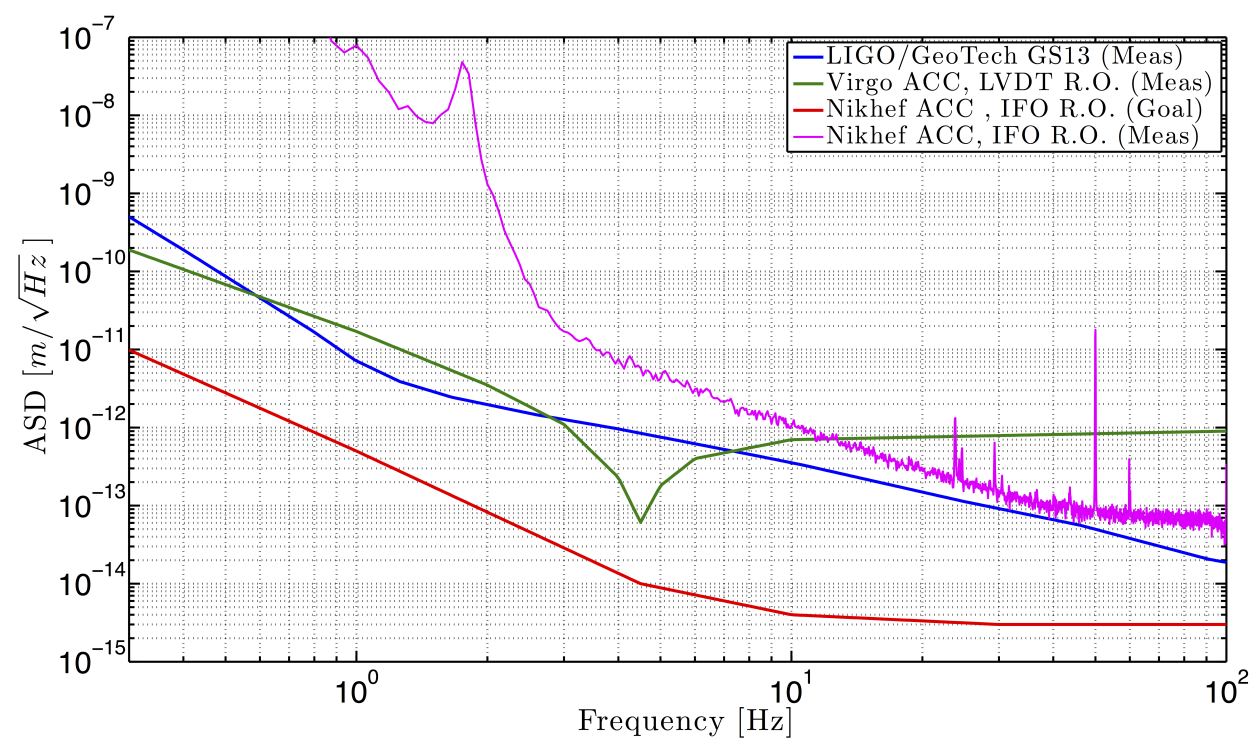

Figure 7: ASD showing a measurement using the VC signal as the sensor's output compared to the goal. Also plotted are two of the best sensors in the field: the Virgo accelerometer, with similar mechanics as this sensor but different readout and the GS13, a geophone in which a suspended mass read out by an inductive velocity measurement

the residual angular motion of the bench will be smaller and the proof mass can be held closer to its lock-point.

The polarization noise can possibly be solved by seismically isolating the platform outside the vacuum tank on which the laser and fiber are housed. The vacuum feedthrough bringing the fiber inside the vacuum tank is the weak spot of this approach; vibrations from the ground couple to the fiber at that point. Another possibility would be to use polarization maintaining (PM) fiber.

\section{References}

[1] A. Bertolini et al., NIM A, 556, pp 616-623 (2006)

[2] H. B. Callen en T. A. Welton, Phys. Rev. 83, 34 (1951)

[3] M.B. Gray et al., Opt.Quant. Electron., 31, pp 571-582 (1999) 